\title{
Timoma: discussão sobre tratamento e prognóstico*
}

\author{
Paulo Manuel PêGo-Fernandes ${ }^{1}$, Gustavo Xavier Ebaid², Maurício Stanzione Galizia², \\ PAUlo MARChiORI ${ }^{3}$, Francisco VARGAS SUSO ${ }^{4}$, FABio BisCEgli JATENE ${ }^{1 *}$
}

Objetivo: Mostrar uma casuística de pacientes com timoma, tratados cirurgicamente, com ou sem outra terapia associada, no Hospital das Clínicas da Faculdade de Medicina da Universidade de São Paulo, São Paulo, Sudeste do Brasil, avaliando o prognóstico destes. Métodos: Entre 1965 e 1998 foram operados 104 pacientes com neoplasias do timo, sendo 69 (66,3\%) do sexo masculino; a idade média ao diagnóstico foi de 47,9 $\pm 16,3$ anos, com faixa de variação de 13 a 76 anos de idade.

Resultados: Dos 104 operados, 89 (85,6\%) foram submetidos a ressecção total do timoma, 6 (5,8\%) a ressecção parcial e $9(8,6 \%)$ a biópsia. O diagnóstico anatomopatológico (timoma x timoma maligno) e a cirurgia (biópsia $\mathrm{x}$ ressecção total $\mathrm{x}$ ressecção parcial) foram significativamente preditivos $(\mathrm{p}<0,02)$ para o tempo médio de sobrevida. Conclusão: A ressecção completa é o tratamento de escolha para os timomas. Esses tumores, quando não invasivos e ressecados completamente, apresentam bom prognóstico imediato e tardio. (J Pneumol 2001;27(6):289-294)

\section{Thymoma: discussion about treatment and diagnosis}

Objectives: To describe a group of patients with thymoma who were submitted to surgical treatment associated or not with therapy, and evaluate their prognosis. Methods: Of the 104 patients with thymic tumors who were operated at Hospital das Clínicas - São Paulo University Medical School between 1965 and 1998, (66.3\%) were males. Mean age at diagnosis, was 47.9\% \pm 16.3 years (ranging from 13 to 76 years). Results: Of the 104 patients, 89 (85.6\%) underwent complete thymoma resection, $6(5.8 \%)$ underwent partial resection and, in $9(8.6 \%)$ of them, biopsy was performed. Anatomopathologic diagnosis (thymoma vs malignant thymoma) and the type of surgery (biopsy vs complete resection vs partial resection) were significant predictors of survival mean time $(\mathrm{p}<0.02)$. Conclusions: Complete resection is the treatment of choice for thymomas. These tumors, when not invasive and completely resected, have good immediate and late prognosis.

Descritores - Timoma.

Key words - Thymoma.

\section{INTRODUÇÃO}

Timoma, um tumor infreqüente e de crescimento lento, é usualmente confinado localmente ao mediastino.

\footnotetext{
* Trabalho realizado no Instituto do Coração do Hospital das Clínicas da Faculdade de Medicina da Universidade de São Paulo, São Paulo, SP.

1. Professor Livre-Docente da Disciplina de Cirurgia Torácica.

2. Acadêmico do $5^{\circ}$ ano.

3. Médico.

4. Professor Titular da Disciplina de Pneumologia.
}

Endereço para correspondência - Prof. Dr. Paulo Manuel Pêgo-Fernandes, Av. Dr. Enéas de Carvalho Aguiar, 44, 2o and., Div. Cirúrgica - InCor - 05403-900 - São Paulo, SP, Brasil. Fax: 3069-5248; Email: paulopego@incor.usp.br

Recebido para publicação em 11/1/01. Aprovado, após revisão, em 1/7/01.

J Pneumol 27(6) - nov-dez de 2001
Localizado em sua porção anterior, é o tumor mais freqüente desse compartimento, compreendendo aproximadamente de 20 a $30 \%$ das massas mediastinais em adultos $^{(1)}$. Os pacientes com timoma podem apresentar quadro clínico não muito específico: tosse, dispnéia, dor torácica, perda de peso, queda do estado geral. Porém, alguns pacientes são diagnosticados por radiografias de tórax em exames de rotina - que mostram opacidade homogênea no mediastino ântero-superior - ou por apresentar miastenia gravis associada ${ }^{(2)}$. Grande parte dos pacientes que apresentam timoma é de portadores de outras doenças auto-imunes ou endócrinas. Cerca de $27 \%$ dos pacientes com miastenia gravis apresentam timoma - 83\% dos homens com miastenia gravis entre 50 e 60 anos possuem timoma ${ }^{(3)}-e 20-40 \%$ dos pacientes com timoma apresentam miastenia gravis. Artrite reumatóide, miosite e aplasia por células vermelhas puras são outras síndromes que podem vir associadas ao timoma ${ }^{(4)}$.

O timoma está mais presente nas $5^{\mathrm{a}}$ e 6a décadas de vida, sendo menos freqüente em pacientes abaixo de 40 
anos de idade. Não há relação com sexo e raça e existe freqüência maior dos timomas malignos sobre os benignos.

A maioria dos timomas é encapsulada, mas aproximadamente $20 \%$ invadem tecido ao redor. Embora esses timomas encapsulados sejam chamados de benignos, são potencialmente invasivos $e$, portanto, seriam considerados um estágio precoce da doença maligna ${ }^{(5)}$. Assim, os timomas podem ser classificados em malignos e benignos, histologicamente de difícil separação, sendo o achado cirúrgico o que realmente define a malignidade: a) invasão local (pulmão, pleura, pericárdio, grandes vasos, diafragma); b) metástases intra ou extratorácicas.

O timoma é derivado do epitélio tímico, apesar de possuir um componente variável de linfócitos ${ }^{(6)}$. Tradicionalmente, a mais comum classificação histológica tem sido baseada na predominância de linfócitos, células epiteliais ou células fusiformes (spindle). Essa classificação está, entretanto, fracamente ligada ao prognóstico ${ }^{(7)}$, já que é baseada no componente linfocítico, que não é essencial à patologia do timoma. Há divergências na literatura quanto à melhor forma de estadiar e classificar essa doença. Os métodos mais importantes são de Masaoka et al. ${ }^{\left({ }^{8}\right)}$ que determina o grau de envolvimento da doença, clínica $e$ histopatologicamente - e do French Study Group on Thymic Tumors (sistema GETT), que é baseado em aspectos cirúrgicos e patológicos do tumor ${ }^{(9)}$.

Ressecção total seguida por radioterapia é o tratamento de escolha para todos os timomas, exceto tumores em estágio IA (de Masaoka), que podem ser tratados somente com cirurgia. Apesar de o timoma ser radiossensível ${ }^{(10)}$, há controvérsias quanto à aplicação de radioterapia em pacientes com timoma invasivo(11). Trabalhos recentes mencionam que a quimioterapia pode melhorar o resultado dos timomas invasivos estágios III e IV (de Masaoka) ou de timomas recorrentes ${ }^{(12)}$.

O objetivo deste trabalho é mostrar uma casuística de 104 pacientes com timoma, tratados cirurgicamente, com ou sem outro tratamento adjuvante, no Hospital das Clínicas da Faculdade de Medicina da Universidade de São Paulo, avaliando o prognóstico destes.

\section{Casuística e mÉtodos}

Entre 1965 e 1998 foram operados 104 pacientes com neoplasias do timo no Hospital das Clínicas da Faculdade de Medicina da Universidade de São Paulo, tendo sido excluídos desta análise os doentes portadores de tumor carcinóide ou neuroendócrino. A idade média no momento do diagnóstico foi de 47,9 $\pm 16,3$ anos, faixa de 13 a 76 anos de idade, sendo $69(66,3 \%)$ indivíduos do sexo masculino. Havia sintomas em $49 \%$ dos doentes, sendo que a maioria apresentava miastenia gravis $(42,3 \%)$. Ape-
Siglas e abreviaturas utilizadas neste trabalho

$\mathrm{F}$ - Feminino

M - Masculino

ASSINT - Assintomático

MG - Miastenia gravis

T MALIG - Timoma maligno

QC - Quadro clínico

DiagAP - Diagnóstico anatomopatológico

CIR - Cirurgia

RT - Radioterapia

QT - Quimioterapia

R PARCIAL - Ressecção parcial

R TOTAL - Ressecção total

TMS - Tempo médio de sobrevida

nas $4(3,8 \%)$ pacientes se apresentaram com síndrome da veia cava superior e $3(2,9 \%)$ com sintomas inespecíficos.

Dos 104 pacientes operados, $89(85,6 \%)$ foram submetidos à ressecção total do timoma, $6(5,8 \%)$ à ressecção parcial e $9(8,6 \%)$ à biópsia.

Apenas 26 doentes (25\%) receberam algum tipo de terapia complementar, sendo realizada radioterapia (RT) em 19 (73\%) casos, quimioterapia (QT) em 2 (7,7\%) e associação de radioterapia e quimioterapia (RT/QT) em 5 (19,3\%).

Para estimar a probabilidade de sobrevida ao longo do tempo utilizou-se o método de Kaplan-Meier ${ }^{(13)}$. Essas estimativas foram obtidas estratificando os doentes de acordo com as variáveis de interesse e a comparação entre os estratos foi feita através do teste de log-rank ${ }^{(13)}$. O teste do escore de log-rank ${ }^{(14)}$ foi utilizado para verificar a relação entre a probabilidade de sobrevida e a idade, que é uma variável quantitativa. Realizou-se análise univariada para as variáveis sexo, idade, quadro clínico (QC), diagnóstico anatomopatológico (DiagAP) e tipo de cirurgia (CIR). As diferenças foram consideradas significativas quando $p$ $<0,05$. As variáveis que mostraram significância na análise univariada foram testadas utilizando-se o modelo multivariado de regressão de $\operatorname{Cox}^{(15)}$. Os cálculos foram feitos por meio do sistema computacional SAS ${ }^{(16,17)}$ (Statistical Analysis System).

\section{RESULTADOS}

Dos 104 pacientes submetidos à operação para ressecção de timoma, 60 (57\%) estavam vivos após um período de acompanhamento médio de 215 meses (variando de 11 meses a 419 meses). Dezessete casos foram perdidos ao longo do seguimento. Nove doentes $(8,6 \%)$ faleceram em virtude da progressão do tumor, 10 (9,6\%) devido a outras intercorrências, como broncopneumonia, leucemia mielóide, miastenia gravis, morte súbita e tuber- 


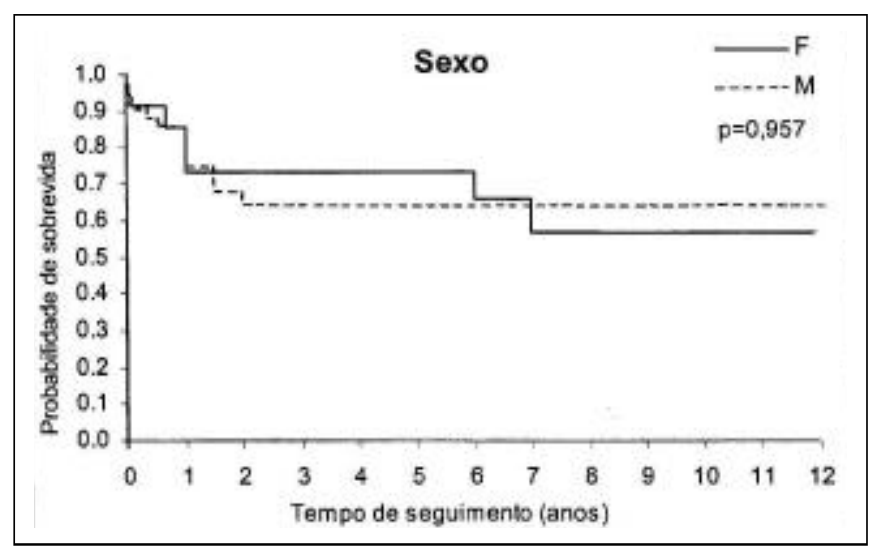

Figura 1 - Estimativa da probabilidade de sobrevida - amostra estratificada por sexo. A taxa de sobrevida, independente do sexo, após um, cinco e 10 anos, respectivamente, é $74,1 \%, 68,7 \%$ e $60,4 \%$.

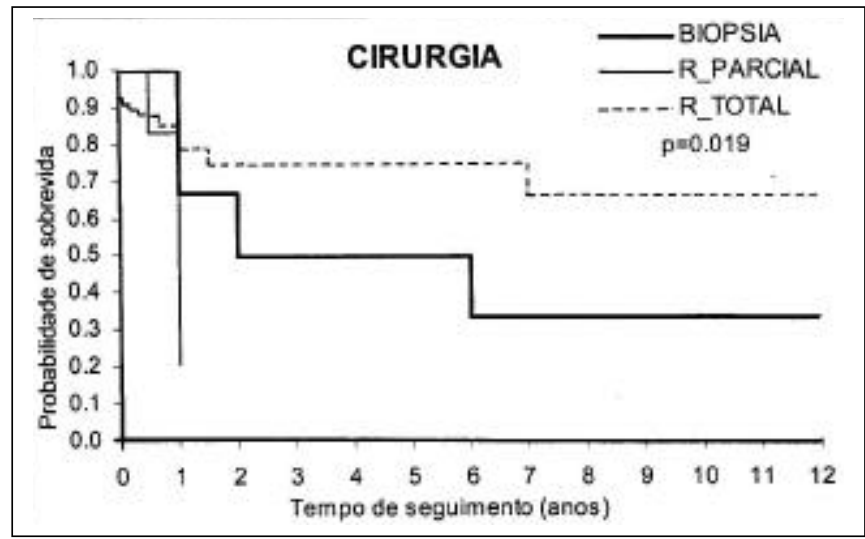

Figura 3 - Taxa de sobrevida relacionada ao tipo de cirurgia: biópsia, cirurgia parcial e cirurgia total.

culose pulmonar e 8 devido a intercorrências não conhecidas. O tempo médio de sobrevida (TMS) foi de $30 \mathrm{me}$ ses. A taxa de sobrevida em um ano foi de $74,1 \%$ (74,9\% para homens e $73,3 \%$ para mulheres), em cinco anos foi de $68,7 \%$ (64,1\% para homens e $73,3 \%$ para mulheres) e em 10 anos foi de 60,4\% (64,1\% para homens e 56,6\% para mulheres) (Figura 1).

Dos 87 pacientes seguidos (desconsiderando os que foram perdidos ao longo do seguimento e os que morreram de intercorrências não conhecidas), 22 (25,28\%) apresentavam timoma maligno e 65 (74,72\%), timoma benigno. Dos pacientes com timoma e timoma maligno diagnosticados, a sobrevida, respectivamente, em um ano foi de $82,6 \%$ e 50,2\%; em cinco anos, de $82,6 \%$ e $33,5 \%$ e em 10 anos, de 82,6\% e 16,7\% (Figura 2).

A maioria dos pacientes $(85,6 \%)$ foi submetida à ressecção total. Em 8,6\% foi feita somente biópsia e 5,8\% foram submetidos à ressecção parcial. Dos pacientes sub-

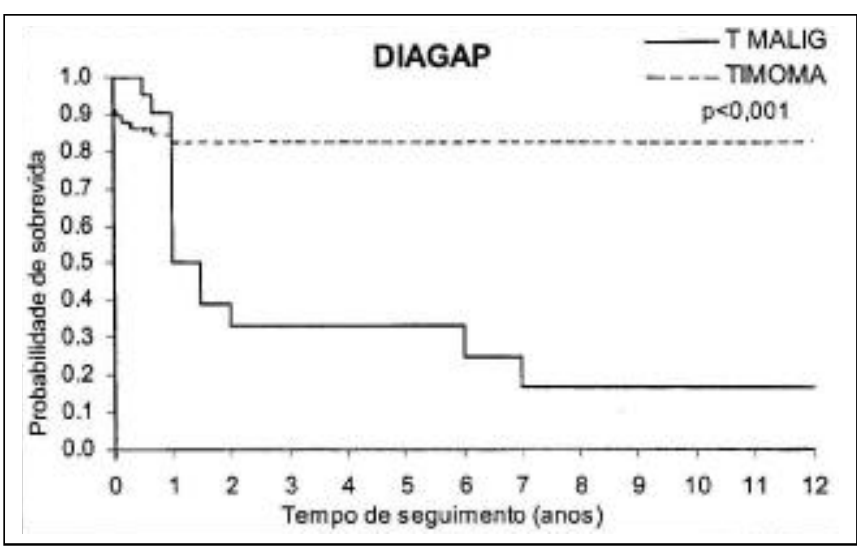

Figura 2 - Estimativa da probabilidade de sobrevida - amostra estratificada por diagnóstico anatomopatológico (DiagAP)

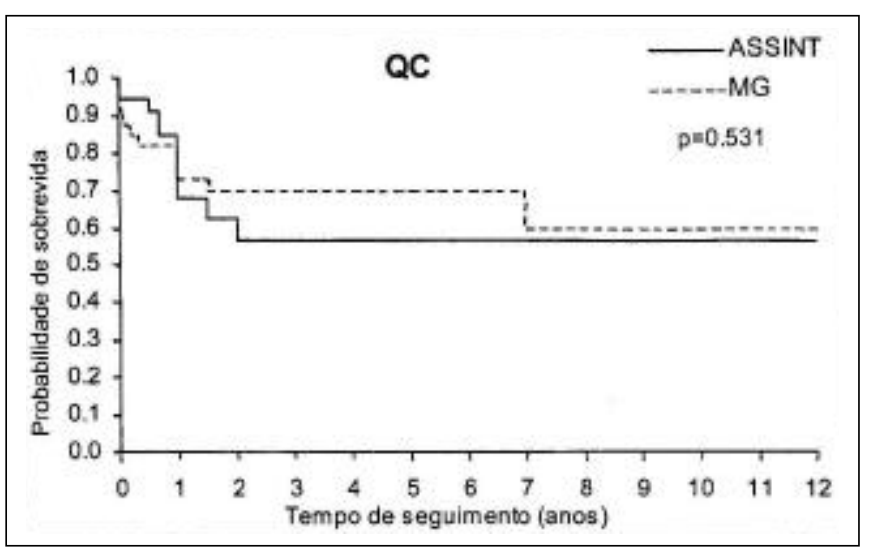

Figura 4 - Taxa de sobrevida relacionada ao quadro clínico: assintomático e miastenia gravis.

metidos à ressecção total, ressecção parcial e biópsia, a sobrevida, respectivamente, em um ano foi de 78,9\%, $20,8 \%$ e 66,7\%; em cinco anos, de 74,6\%, 0,0\% e $50 \%$ e, em 10 anos, de 66,3\%, 0,0\% e 33,3\% (Figura 3).

Quanto ao quadro clínico, $39 \%$ eram assintomáticos, $44,8 \%$ apresentavam miastenia gravis e $16,2 \%$, sintomas inespecíficos. Dos pacientes assintomáticos e com miastenia gravis, a sobrevida, respectivamente, em um ano foi de $67,5 \%$ e $73,3 \%$; em cinco anos, de $56,7 \%$ e $69,6 \%$ e, em 10 anos, de 56,7\% e 59,6\% (Figura 4).

Dos 76 pacientes submetidos a ressecção completa sem radioterapia (RT) adjuvante, $3(3,9 \%)$ morreram por progressão local do tumor. Dos que receberam RT e tiveram ressecção total (12 pacientes), nenhum morreu pelo tumor.

Entre os pacientes que foram submetidos a ressecção parcial (seis pacientes), 4 (67\%) morreram pelo tumor apesar de terem recebido RT adjuvante. Entre os pacientes que fizeram apenas biópsia (nove pacientes), 6 (67\%) receberam RT. Dentre estes, 2 (33\%) morreram pelo tumor. 
Quinze pacientes receberam RT pós-operatória, cinco deles com quimioterapia (QT) concomitante. Dois pacientes receberam apenas $\mathrm{QT}$.

Dos 23 pacientes com DiagAP de timoma maligno, 16 (70\%) receberam RT pós-operatória. Destes, 7 (44\%) morreram pela progressão do tumor, dos quais, $1(14 \%)$ tinha feito ressecção total, 4 (57\%) ressecção parcial e 2 (29\%) apenas biópsia. Dos 81 pacientes com DiagAP de timoma, $4(5 \%)$ receberam RT pós-operatória. Dentre esses pacientes submetidos a ressecção completa, não houve óbitos relacionados à neoplasia.

\section{Fatores prognósticos}

DiagAP (timoma $\mathrm{x}$ timoma maligno) e cirurgia (biópsia $\mathrm{x}$ ressecção total $x$ ressecção parcial) foram significativamente preditivos $(\mathrm{p}<0,02)$ para o TMS. Sexo, quadro clínico e idade não influenciaram significativamente o TMS (Tabela 1).

As variáveis que se mostraram significativas na análise univariada foram também testadas pelo modelo de regressão de Cox para estabelecer significância independente (Tabela 2). O DiagAP foi o fator mais preditivo para o TMS (razão de risco $=3,961 ; \mathrm{p}<0,001$ ) quando comparado com a variável cirurgia.

\section{DISCUSSÃO}

Timoma, uma doença rara do epitélio tímico, é a mais comum neoplasia primária do timo. A taxa média de sobrevida em alguns estudos é de $67 \%$ em cinco anos e $53 \%$ em 10 anos $^{(7)}$ - em nosso estudo foi de $68 \%$ em

TABELA 1

Fatores prognósticos

\begin{tabular}{lc}
\hline Variável & Teste de log rank $(\mathbf{p})$ \\
\hline Sexo & 0,957 \\
QC & 0,531 \\
DiagAP & $<0,001^{*}$ \\
Cirurgia & $0,019^{*}$ \\
Idade & 0,900 \\
\hline
\end{tabular}

QC - Quadro clínico; DiagAP - Diagnóstico anatomopatológico

\section{TABELA 2}

Análise multivariada para o fator mais preditivo para o tempo médio de sobrevida

\begin{tabular}{lccccc}
\hline Variável & Estimativa & $\begin{array}{c}\text { Erro } \\
\text { padrão }\end{array}$ & $\mathbf{p}$ & $\begin{array}{c}\text { Razão } \\
\text { de risco }\end{array}$ & IC \\
\hline DiagAP & 1,376 & 0,393 & $<0,001$ & 3,961 & $1,831-8,567$ \\
\hline
\end{tabular}

p- nível descritivo do teste do parâmetro ser nulo; IC - intervalo com 95\% de confiança para a razão de risco cinco anos e de 60,4\% em 10 anos. Timoma segue normalmente curso indolente e os sintomas ocorrem em somente metade dos $\operatorname{casos}^{(4)}$.

A definição de fatores prognósticos é essencial para identificar grupos de pacientes que podem beneficiar-se da ressecção radical. Entretanto, é difícil definir fatores prognósticos para timoma, já que estes tumores são incomuns, polimórficos e de crescimento lento. Portanto, grande quantidade de pacientes deve ser estudada, por um período longo, para análise dos fatores prognósticos.

Mistenia gravis (MG), a doença auto-imune mais freqüentemente associada ao timoma, ocorre em aproximadamente $30 \%$ dos pacientes ${ }^{(18)}$. O valor prognóstico da MG é controverso. Afirma-se que pacientes com MG têm pior prognóstico(19). A maior taxa de óbito foi atribuída à mortalidade perioperatória elevada. Entretanto, em concordância com alguns trabalhos recentes ${ }^{(7,20-23)}$, não encontramos nenhuma influência estatisticamente considerável da MG na sobrevida dos pacientes com timoma. Avanços no tratamento médico provavelmente contribuíram para vencer as complicações ${ }^{(24)}$; por outro lado, MG pode facilitar o diagnóstico precoce, resultando em maior sobrevi$\mathrm{da}^{(22)}$.

Os timomas podem ser classificados de acordo com seu tipo histológico ou com seu grau de invasão. Histologicamente, são classificados de acordo com a predominância de células epiteliais ou linfocíticas (linfocítico, epitelial, misto ou spindle), sistema proposto por Bernatz et al. ${ }^{(25)}$, ou pela semelhança morfológica com o epitélio cortical ou medular. Há constantes evidências de que os tipos linfocítico e spindle têm melhor prognóstico, tendo sido encontradas taxas de sobrevida de até $90 \%$ em 10 $\operatorname{anos}^{(26,27)}$. Já o tipo epitelial apresenta prognóstico pior, tendo sido relatadas taxas de sobrevida em torno de $30 \%$ em cinco anos ${ }^{(4,7,26)}$. Infelizmente, ocorre grande variação na composição celular tumoral e não há relação consistente entre a aparência microscópica e o comportamento biológico dessas neoplasias ${ }^{(28)}$. Desse modo, não realizamos tal classificação neste estudo.

Do ponto de vista da invasão, os timomas são classificados em malignos ou benignos, de acordo com a presença de invasão macroscópica de estruturas adjacentes, metástases ou evidência microscópica de invasão capsular ${ }^{(28)}$. A maioria dos timomas comporta-se de maneira benigna ${ }^{(29)}$, tendo sido descritas incidências de 15 a $65 \%{ }^{(28)}$.

A invasão tumoral é tida como um fator prognóstico muito importante da sobrevida em pacientes portadores de timoma ${ }^{(30)}$. Dentre os métodos existentes para mensurar o grau de invasão do tumor, o sistema classificatório proposto por Masaoka et al. ${ }^{(8)}$ é considerado por muitos autores o fator prognóstico mais importante $e^{(21-23,31)}$.

Vários autores ${ }^{(7,32-36)}$ têm enfatizado o valor prognóstico de um novo método histopatológico de classificação 
dos timomas, proposto por Marino e Müller-Hermelink ${ }^{(37)}$, mas a superioridade de sua classificação não foi ainda confirmada ${ }^{(38,39)}$.

Nossa série de pacientes mostrou-se em concordância com os principais estudos já discutidos. O tumor foi classificado como maligno ou não-maligno. $\mathrm{O}$ tipo benigno apresentou sobrevida em 10 anos de $82,6 \%$, enquanto o maligno mostrou sobrevida em cinco anos de $33,5 \%$. A maioria dos tumores encontrados era benigna $(74,7 \%) e$ esta classificação quanto ao grau da invasão foi significativamente preditiva para a sobrevida média $(\mathrm{p}<0,02)$.

Cirurgia é o tratamento de escolha para pacientes com timoma não metastático, independentemente de sua in-

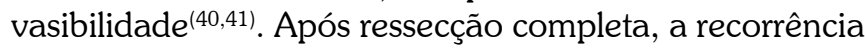
localizada de timoma encapsulado só ocorre excepcionalmente $e^{(42,43)}$, com prognóstico excelente, tendo sido descritas taxas de sobrevida de mais de $90 \%$ em cinco anos ${ }^{(8,19)}$. A melhor técnica de exposição operatória é obtida utilizando-se esternotomia mediana ${ }^{(28)}$. Foi demonstrado que mais de $90 \%$ dos pacientes operados não apresentam nenhuma complicação pós-operatória e que a taxa de mortalidade pós-operatória não é influenciada pela $M G^{(52)}$.

Em nossa série de pacientes, a grande maioria (86\%) foi submetida à ressecção total do tumor, sendo que 76 não receberam nenhuma terapia adjuvante. Entre esses pacientes, apenas 3,9\% morreram pelo tumor, o que está de acordo com as altas taxas de sobrevida encontradas na literatura. $\mathrm{Na}$ análise univariada, o tipo de cirurgia realizada mostrou ser um fator prognóstico significativamente preditivo para o tempo médio de sobrevida (TMS). No entanto, o tipo de cirurgia realizada poderia apenas estar refletindo o grau de malignidade do tumor no momento da intervenção. Essa hipótese se fortalece ao utilizarmos o método de regressão de Cox para estabelecer significância independente. Nessa análise, o diagnóstico anatomopatológico (tumor maligno $\mathrm{x}$ tumor benigno) mostrouse fator mais preditivo quando comparado com o tipo de cirurgia.

\section{REFERÊNCIAS}

1. Levine GD, Rosai J. Thymic hyperplasia and neoplasia: a review of current concepts. Hum Pathol 1978;9:495-515.

2. Arriagada R, Gerard-Marchant R, Tubiana M, et al. Radiation therapy in the management of malignant thymic tumors. Acta Radiol Oncol 1981;20:167-172.

3. Monden Y, Nakahara K, Kagotani K, et al. Myasthenia gravis with thymoma: analysis of an postoperative prognosis for 65 patients with thymomatous myasthenia gravis. Ann Thorac Surg 1984;38:46-52.

4. Gripp S, Hilgers K, Wurm R, et al. Thymoma: prognostic factors and treatment outcomes. Cancer 1998;83:1495-1503.

5. Bergh NP, Gatzinsky P, Larsson S, et al. Tumors of the thymus and thymic region. I. Clinicopathological studies on thymomas. Ann Thorac Surg 1978;25:91-98.
Em casos de disseminação pleural, radioterapia como forma única de tratamento é efetiva ${ }^{(44)}$. Irradiação préoperatória tem sido empregada, com sucesso, para síndrome da veia cava superior e para facilitar cirurgia subseqüente ${ }^{(45,46)}$. Entretanto, a radioterapia é normalmente administrada no pós-operatório e é recomendada, em timomas invasivos, para reduzir sua recorrência ${ }^{(21,22,47)}$. Com radioterapia pós-operatória, a sobrevida após ressecção completa de um timoma invasivo é aproximada à de pacientes com tumor não invasivo ${ }^{(48)}$.

A recidiva após ressecção completa de um timoma invasivo é de aproximadamente $20 \%$, enquanto menos que $5 \%$ desenvolvem metástase extratorácica ${ }^{(49)}$. Assim, terapia agressiva local, neste caso a radioterapia, é necessária. Essa terapia reduz a freqüência da falência local após ressecção completa de timoma invasivo de $28 \%$ para $5 \%$. Logo, a ressecção isolada tem sido considerada terapia insuficiente para timoma invasivo e a radioterapia pósoperatória é recomendada para todos os timomas invasivos, independente da extensão cirúrgica ${ }^{(50)}$. A freqüência de recidiva local no mediastino foi reduzida de $17,2 \%$ para $3,6 \%{ }^{(51)}$ com a radioterapia.

Alguns autores ${ }^{(18,48)}$, entretanto, recomendam radioterapia pós-operatória para todos os timomas; outros, porém, somente não a indicam para aqueles completamente encapsulados macroscopicamente e sem invasão capsular microscopicamente $e^{(9,52)}$, que podem ser tratados apenas com cirurgia.

Devido ao pequeno número de timomas ressecados completamente e irradiados posteriormente neste estudo (sete pacientes), recomendações definitivas a respeito da situação adjuvante não podem ser obtidas deste subgrupo. Entretanto, com base nas observações da literatura, a radioterapia pós-operatória é recomendável após ressecção completa.

Em conclusão, a ressecção completa é o tratamento de escolha para os timomas. Esses tumores, quando não invasivos e ressecados completamente, apresentam bom prognóstico imediato e tardio.

6. Lauriola L, Maggiano N, Marino M, et al. Human thymoma: immunologic characteristics of the lymphocytic component. Cancer 1981;48: 1992-1995.

7. Lewis JE, Wick MR, Scheithauer BW, et al. Thymoma: a clinicopathologic review. Cancer 1987;60:2727-2743.

8. Masaoka A, Monden Y, Nakahara K, et al. Follow-up study of thymomas with special reference to their clinical stages. Cancer 1981;48: 2485-2492.

9. Cowen D, Hannoun Levi JM, Resbeut M, et al. Natural history and treatment of malignant thymoma. Oncology 1998;12:1001-1005.

10. Ohara K, Okumura T, Sugahara S, et al. The role of preoperative radiotherapy for invasive thymoma. Acta Oncol 1990;29:525-529. 
11. Gonzalez-Gonzalez D. The need for prospective clinical studies in thymomas. Radiother Oncol 1991;21:75-76.

12. Komaki R, Putnam JB Jr, Shin DM, et al. Thymic neoplasms. Curr Opin Oncol 1997;9:156-160.

13. Lee ET. Statistical methods for survival data analysis. California: Lifetime Learning Publications, 1980;75-130.

14. SAS Institute Inc. SAS/STAT ${ }^{\circledR}$ User's Guide, version 6 , $4^{\text {th }}$ ed, vol. 2. Cary, NC: SAS Institute Inc., 1989;1046-1048.

15. Lee ET. Statistical methods for survival data analysis. California: Lifetime Learning Publications, 1980;306-318.

16. SAS Institute Inc. SAS/STAT ${ }^{\circledR}$ User's Guide, version 6, $4^{\text {th }}$ ed, vol. 1 Cary, NC: SAS Institute Inc., 1989.

17. SAS Institute Inc. SAS/STAT ${ }^{\circledR}$ User's Guide, version 6 , $4^{\text {th }}$ ed, vol. 2. Cary, NC: SAS Institute Inc., 1989.

18. Monden Y, Nakahara K, lioka S, et al. Recurrence of thymoma: clinicopathological features, therapy, and prognosis. Ann Thorac Surg 1985;39:165-169.

19. Appelqvist $\mathrm{P}$, Kostiainen $\mathrm{S}$, Franssila K, et al. Treatment and prognosis of thymoma: a review of 25 cases. J Surg Oncol 1982;20:265-268.

20. Cooper JD. Current therapy for thymoma. Chest 1993;103(Suppl): 334S-336S.

21. Wilkins EW Jr, Grillo HC, Scanell JG, et al. Role of staging in prognosis and management of thymoma. Ann Thorac Surg 1991;51:888892.

22. Maggi G, Casadio C, Cavallo A, et al. Thymoma: results of 241 operated cases. Ann Thorac Surg 1991;51:152-156.

23. Shamji F, Pearson FG, Todd TRJ, et al. Results of surgical treatment for thymoma. J Thorac Cardiovasc Surg 1984;87:43-47.

24. Crucitti F, Doglietto GB, Bellantone R, et al. Effects of surgical treatment in thymoma with myasthenia gravis: our experience in $103 \mathrm{pa}$ tients. J Surg Oncol 1992;50:43-46.

25. Bernatz PE, Harrison EJ, Clagett TO. Thymoma: a clinicopathologic study. J Thorac Cardiovasc Surg 1961;42:424-444.

26. Blumberg D, Port J, Weksler B, et al. Thymoma: a multivariate analysis of factors predicting survival. Ann Thorac Surg 1995;60:908-914.

27. Urgesi A, Monetti U, Rossi G, et al. Aggressive treatment of intrathoracic recurrences of thymoma. Radiother Oncol 1992;24:221-225.

28. Davis RD, Sabiston DC. The mediastinum. In: Sabiston Jr DC, Lyerly HK, eds. Textbook of surgery: the biological basis of modern surgical practice. $15^{\text {th }}$ ed. Philadelphia: W.B. Saunders, 1997.

29. Ibrahim NB, Briggs JC, Jeyasingham K, et al. Metastasizing thymoma. Thorax 1982;37:771-773.

30. Pich A, Chiarle R, Chiusa L, et al. Long-term survival of thymoma patients by histologic pattern and proliferative activity. Am J Surg Pathol 1995;19:918-926.

31. Pescarmona E, Rendina EA, Venuta F, et al. Analysis of prognostic factors and clinicopathological staging of thymoma. Ann Thorac Surg 1990;50:534-538.

32. Morgenthaler TI, Brown LR, Colby TV, et al. Thymoma. Mayo Clin Proc 1993; 68:1110-1123.
33. Pescarmona E, Rendina EA, Venuta F, et al. The prognostic implication of thymoma histologic subtyping: a study of 80 consecutive cases. Am J Clin Pathol 1990;93:190-195.

34. Elert O, Buchwald J, Wolf K. Epithelial thymus tumors: therapy and prognosis. J Thorac Cardiovasc Surg 1988;36:109-113.

35. Engel P, Pilsgaard B, Francis D. Thymomas and thymic carcinomas: a retrospective investigation with histological reclassification. APMIS 1995; 103:671-678.

36. Tan PH, Sng ITY. Thymoma: a study of 60 cases in Singapore. Histopathology 1995;26:509-518.

37. Marino M, Müller-Hermelink HK. Thymoma and thymic carcinoma: relation of thymoma epithelial cells to the cortical and medullary differentiation of thymus. Virchows Arch A Pathol Anat Histopathol 1985; 407:119-149.

38. Pan CC, Wu HP, Yang CF, et al. The clinicopathological correlation of epithelial subtyping in thymoma: a study of 112 consecutive cases. Hum Pathol 1994;25:893-899.

39. Kornstein MJ, Curran WJ Jr, Turrisi AT III, Brooks JJ. Cortical versus medullary thymomas: a useful morphologic distinction? Hum Pathol 1988; 19:1335-1339.

40. Maggi G, Giaccone G, Donadio M, et al. Thymomas: a review of 169 cases, with particular reference to results of surgical treatment. Cancer 1986;58:765-776

41. Wilkins EW. Thymoma: surgical treatment. In: Wood DE, Thomas CR, ed. Mediastinal tumors. Berlin: Springer, 1995;11-18.

42. Curran WJ Jr, Kornstein MJ, Brooks JJ, et al. III. Invasive thymoma: the role of mediastinal irradiation following complete or incomplete surgical resection. J Clin Oncol 1988;6:1722-1727.

43. Ruffini E, Mancuso M, Oliaro A, et al. Recurrence of thymoma: analysis of clinicopathologic features, treatment, and outcome. J Thorac Cardiovasc Surg 1997;113:55-63.

44. Ichinose $Y$, Ohta M, Yano $T$, et al. Treatment of invasive thymoma with pleural dissemination. J Surg Oncol 1993;54:180-183.

45. Fugimura $\mathrm{S}$, Kondo $\mathrm{T}$, Handa $\mathrm{M}$, et al. Results of surgical treatment for thymoma based on 66 patients. J Thorac Cardiovasc Surg 1987;93: 708-714.

46. Ribet M. Preoperative radiotherapy for lymphoepithelial thymomas. Ann Chir 1993;47:766-768.

47. Haniuda M, Morimoto $M$, Nishimura $\mathrm{H}$, et al. Adjuvant radiotherapy after complete resection of thymoma. Ann Thorac Surg 1992;54:311315.

48. Nakahara K, Ohno K, Hashimoto J, et al. Thymoma: results with complete resection and adjuvant postoperative irradiation in 141 consecutive patients. J Thorac Cardiovasc Surg 1988;95:1041-1047.

49. Jackson MA, Ball DL. Postoperative radiotherapy in invasive thymoma. Radiother Oncol 1991;21:77-82.

50. Hara N, Yoshida T, Furukawa T, et al. Thymoma: clinicopathologic features, therapy, and prognosis. Jpn J Surg 1980;10:232-327.

51. Urgesi A, Monetti U, Rossi G, et al. Role of radiation therapy in locally advanced thymoma. Radiother Oncol 1990;19:273-280.

52. Regnard JF, Magdeleinat P, Dromer C, et al. Prognostic factors and long-term results after thymoma resection. A series of 307 patients. $1996 ; 112 \cdot 376-384$ 